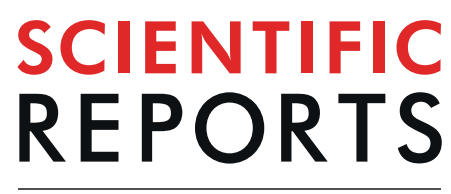

\title{
An experiment-informed signal transduction model for the role of the Staphylococcus aureus MecR1 protein in $\beta$-lactam resistance
}

\author{
Bruno S. Belluzo ${ }^{1,4}$, Luciano A. Abriata $\mathbb{1}^{2,4}$, Estefanía Giannini ${ }^{1,4}$, Damila Mihovilcevic ${ }^{1}$, \\ Matteo Dal Peraro ${ }^{2}$ \& Leticia I. Llarrull $\mathbb{1}^{1,3 *}$
}

The treatment of hospital- and community-associated infections by methicillin-resistant Staphylococcus aureus (MRSA) is a perpetual challenge. This Gram-positive bacterium is resistant specifically to $\beta$-lactam antibiotics, and generally to many other antibacterial agents. Its resistance mechanisms to $\beta$-lactam antibiotics are activated only when the bacterium encounters a $\beta$-lactam. This activation is regulated by the transmembrane sensor/signal transducer proteins BlaR1 and MecR1. Neither the transmembrane/metalloprotease domain, nor the complete MecR1 and BlaR1 proteins, are isolatable for mechanistic study. Here we propose a model for full-length MecR1 based on homology modeling, residue coevolution data, a new extensive experimental mapping of transmembrane topology, partial structures, molecular simulations, and available NMR data. Our model defines the metalloprotease domain as a hydrophilic transmembrane chamber effectively sealed by the aposensor domain. It proposes that the amphipathic helices inserted into the gluzincin domain constitute the route for transmission of the $\beta$-lactam-binding event in the extracellular sensor domain, to the intracellular and membrane-embedded zinc-containing active site. From here, we discuss possible routes for subsequent activation of proteolytic action. This study provides the first coherent model of the structure of MecR1, opening routes for future functional investigations on how $\beta$-lactam binding culminates in the proteolytic degradation of Mecl.

Methicillin-resistant Staphylococcus aureus (MRSA) is a huge challenge with respect to the control of hospitaland community-associated infection ${ }^{1-7}$. This bacterium is generally resistant to several antibacterial agents, and especially resistant to the $\beta$-lactam antibiotics ${ }^{8}$. Few new antibacterials are in advanced stages of clinical evaluation for the treatment of MRSA infection ${ }^{9-13}$ and resistant MRSA strains evolve rapidly ${ }^{14-16}$. Resistance to $\beta$-lactam antibiotics in MRSA is inducible ${ }^{17,18}$ and is the result of the expression of two resistance enzymes. One is the PC1 $\beta$-lactamase (BlaZ) ${ }^{19}$ and the second is the PBP2a transpeptidase. The causative event for the bactericidal mechanism of the $\beta$-lactams is inactivation of the critical PBP-catalyzed crosslinking of the peptidoglycan cell wall of the bacterium. In contrast to the other PBPs of S. aureus, PBP2a has low affinity for almost all $\beta$-lactam antibiotics, and hence is not inhibited by the concentrations of $\beta$-lactams that are achievable clinically ${ }^{20}$. The genes that code for these two resistance determinants are part of the bla and mec operons, respectively ${ }^{21,22}$. These operons also include the genes for a transmembrane sensor/transducer protein (BlaR1- $\beta$-lactam antibiotic receptor protein - and MecR1-methicillin receptor protein-, respectively) and a DNA-binding protein (BlaI and MecI, respectively). The mec operon also includes an additional protein, MecR2, which is an antirepressor ${ }^{23}$. The regulation of the expression of these resistance determinants by BlaR1 and MecR1 have strong mechanistic parallel: they both detect the presence of $\beta$-lactam antibiotics in the milieu using an extracellular sensor domain, by catalysis of an irreversible acylation of an active site serine by the $\beta$-lactam. This acylation is transduced to the cytoplasmic

${ }^{1}$ Instituto de Biología Molecular y Celular de Rosario (IBR, CONICET-UNR), Predio CONICET Rosario, 27 de Febrero 210 bis, 2000, Rosario, Argentina. ${ }^{2}$ Laboratory for Biomolecular Modeling - École Polytechnique Fédérale de Lausanne and Swiss Institute of Bioinformatics, CH-1015, Lausanne, Switzerland. ${ }^{3}$ Área Biofísica, Facultad de Ciencias Bioquímicas y Farmacéuticas, Universidad Nacional de Rosario, Suipacha 570, 2000, Rosario, Argentina. ${ }^{4}$ These authors contributed equally: Bruno S. Belluzo, Luciano A. Abriata and Estefanía Giannini. *email: Ilarrull@ ibr-conicet.gov.ar 


\begin{tabular}{|c|c|c|c|c|c|c|}
\hline & & TopPred & HMMTOP & TMHМM & TMpred & DAS \\
\hline \multicolumn{7}{|c|}{ MecR1 Staphylococcus aureus NRS70 } \\
\hline \multirow{6}{*}{ TM regions } & 1 & $3-23$ & $6-23$ & $5-27$ & $5-28$ & $7-27$ \\
\hline & 2 & $37-57$ & $40-57$ & $40-62$ & $37-55$ & $39-54$ \\
\hline & 3 & $105-125$ & $105-122$ & $103-125$ & $105-125$ & $106-125$ \\
\hline & 4 & $172-192$ & $162-179$ & - & $173-193$ & - \\
\hline & 5 & $216-236$ & - & - & $214-236$ & $220-228$ \\
\hline & 6 & $316-336$ & - & $316-338$ & $316-334$ & $319-330$ \\
\hline
\end{tabular}

Table 1. Topology prediction of MecR1 from Staphylococcus aureus NRS70. Five distinct algorithms were used: TopPred, HMMTOP, TMHMM, TMpred and DAS, all available in www.expasy.org. Six transmembrane (TM) helical regions were found, with different confidence score levels regarding each algorithm. The numbers in black show the amino acid position in which the TM is located. Numbers in red correspond to TM helical regions predicted with a low score (below the cutoff stipulated in each algorithm).

domain as a structural reorganization of the entire protein ${ }^{24,25}$. Neither the nature of this structural reorganization, nor its connection to control of protein expression, is known. The presence of an HEXXH motif in the cytoplasmic domain - a motif that is characteristic of a metalloproteinase ${ }^{26}$-is the center of current hypotheses. The two conserved His residues of this motif are suggested to provide two of the ligands to an active site $\mathrm{Zn}$ (II) atom $^{27}$. Studies on BlaR1 from Bacillus licheniformis identified a glutamic acid as the third zinc-binding ligand ${ }^{28}$. If correct, these structural features place the metalloprotease domain in the gluzincin family ${ }^{29}$. Although changes in the secondary structure of the $S$. aureus proteins are seen using circular dichroism (CD) and Fourier-transform Infrared spectroscopy (FTIR) assays ${ }^{24,30}$, these changes are not evident in the X-Ray structures of the solubilized, apo-sensor domain and $\beta$-lactam-bound sensor domain ${ }^{31,32}$. BlaR1 has proven metalloprotease activity, as it catalyzes proteolysis of BlaI and/or undergoes autoproteolysis ${ }^{19,27}$. BlaI binds to the operator region of the blaR operon, and when bound represses transcription to a low basal level ${ }^{33}$. Loss of BlaI, such as occurs by proteolysis, results in BlaZ expression resulting in $\beta$-lactam resistance ${ }^{27}$. In contrast, no direct evidence implicates MecR1 proteolysis of MecI, although this event is credible. Full activation of the mec operon requires the presence of MecR2. MecR2 binds to MecI and presumably promotes MecI degradation mediated by native cytoplasmatic proteases ${ }^{23}$. Hence, MecR2 interferes with interaction of MecI with the mecA promoter.

Our understanding of the activation of BlaR1 and MecR1 by $\beta$-lactams is limited by the absence of X-Ray structures of the full proteins. We report a combined computational and experimental study that unveils the architecture of MecR1. Our model proposes a hydrophilic transmembrane chamber for the metalloprotease domain, wherein this domain interacts both with the sensor domain through an amphipathic helix and a "reentrant" helix. This reentrant helix is poised to propagate structural perturbation to the zinc site. This model allows us to formulate a proposal for the molecular route by which $\beta$-lactam acylation of the extracellular domain results in the activation of the cytoplasmic metalloprotease domain.

\section{Results}

Coevolution-based methods and homology modeling independently predict a gluzincin-like active site embedded in a transmembrane supermotif. The sensor proteins BlaR1 and MecR1 present an N-terminal metalloprotease domain (residues 1-338) and a C-terminal sensor domain (339-585) ${ }^{24,25}$. Analysis of MecR1 from S. aureus N315 (NRS70) using different algorithms predict three transmembrane (TM) segments in the N-terminal region (residues 1-130; Table 1), and most algorithms additionally predict a fourth TM helix before the C-terminal sensor domain (around residues 316-338). Some algorithms identify two additional lowscore hydrophobic segments in the region that has historically been considered the cytoplasmic domain (residues 126-315). BLAST searches of the MecR1 metalloprotease domain against the Protein Data Bank returned few entries, all of low similarity. Thus, the homology modeling servers RoBETTA, I-TASSER, and Swiss-ModeL find only four proteins as templates for molecular modeling, covering only $\sim 40-60 \%$ of the query sequences and with an identity of only 8.4 to $17.7 \%$. These structures (PDB IDs 4AW6, 4IL3, 2YPT, and 5SYT) are gluzincin motifs embedded in helical transmembrane domains that define an internal hydrophilic chamber ${ }^{34,35}$. The gluzincin fold was consistently transferred by the four programs to the metalloprotease domain (residues 130-300) of MecR1. Coevolution-based modeling with EVFoLD ${ }^{36}$ independently predicted a similar structure for region 130-300, thus supporting the models built from distant homology. In particular, one homology model returned by Roвetta (Model 1) matched very well the coevolution data (Figs. 1a and S1). The convergent consensus emerged from these independent modeling efforts was robust enough to test the resulting model experimentally. Hitherto, our homology- and coevolution-based models all indicated that (i) the gluzincin domain is embedded in a transmembrane helical domain similar to the gluzincin domain of the Ste24p and ZMPSTE24 proteases, and (ii) that one of the potential helices predicted by TOPPRED, HMMTOP and TMPRED (around 172-193, the fourth helix) folds as a small helix and three $\beta$-strands as part of the gluzincin domain. We exploited these similarities to the Ste24p and ZMPSTE24 proteases to further model residues 1-330.

The metalloprotease domain of MecR1 defines a membrane-embedded hydrophilic chamber. The top two templates used by RoBeTTA for the 1-340 sequence of MecR1 were PDB IDs 4AW6 and 2YPT, two X-ray structures of the human nuclear membrane zinc metalloprotease ZMPSTE24 (FACE1) ${ }^{34}$. In ZMPSTE24, seven long transmembrane helices (including two of the gluzincin domain) and one amphipathic 
a

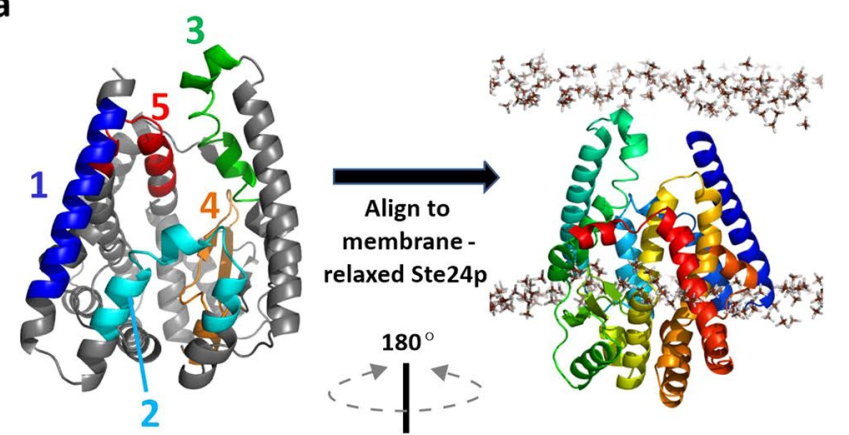

Model 1

Model 1

b

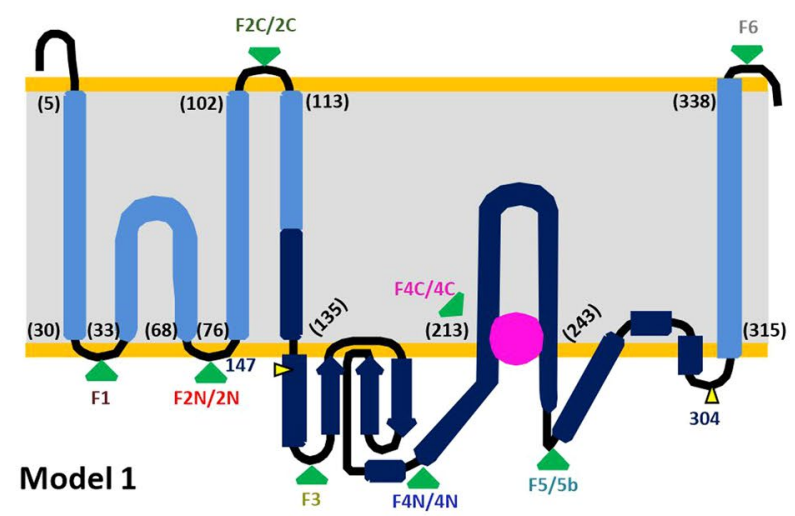

Model 1

C
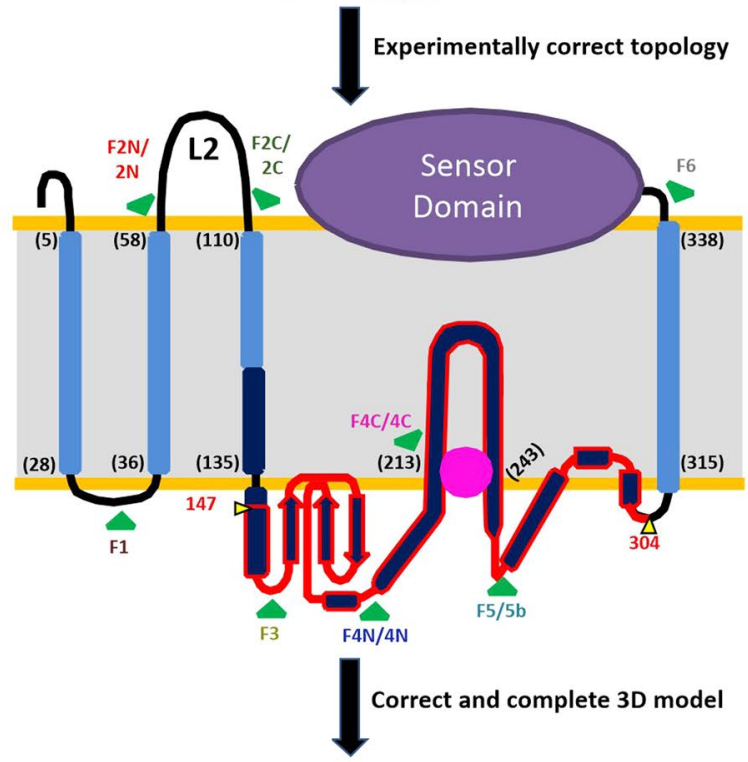

Model 2

Figure 1. (a) Robetta model (Model 1) of residues 1-340 of the metalloprotease domain of MecR1 (cartoon). The left half maps each of the five transmembrane helices predicted from sequence (Table 1), colored from blue (N-terminal) to red (C-terminal). The right half depicts this model aligned to a membrane-relaxed structure of the Ste24p membrane metalloprotease (see Fig. S2). (b) Flat scheme showing the overall topology of the metalloprotease domain of MecR1 according to Model 1; light and dark blue for the whole homology model, dark blue for the portion backed up by coevolution data. (c) Flat scheme showing the overall topology of fulllength MecR1 according to Model 2, which contemplates the results from the experimental mapping of the transmembrane topology. The gluzincin core of MecR1 (MecR1 ${ }^{\mathrm{GLZ}}$, residues 147-304) is shown in dark blue with red border. In $(\mathbf{b}, \mathbf{c})$, the numbers between brackets indicate the amino acid residue where each membraneembedded helix is proposed to start or finish; the pink sphere approximates the position of the zinc ion; the green arrows labelled F1, F2N/2N, F2C/2C, F3, F4N/4N, F4C/4C, F5/5b and F6 indicate the positions where either eGFP was fused or a TEV recognition site was inserted for the experimental topology mapping. 
helix close access to the hydrophilic chamber on the outer membrane side. We evaluated how this protein was accommodated in a membrane. Using coarse-grained and atomistic molecular dynamics simulations, we computationally relaxed the structure of the homologous protein Ste24p from Saccharomyces mikatae (PDB ID 4IL3, a higher resolution structure with fewer missing residues compared to the alternative structures) ${ }^{35}$ in a POPE membrane. This relaxation led to a match of the hydrophobic surface of Ste24p with the hydrophobic interior of the membrane, with the hydrophilic protein surface protruding on both sides of the membrane (Fig. S2). Fitting Model 1 for the metalloprotease domain of MecR1 to our membrane-equilibrated Ste24p (Fig. 1a) leads to two key observations. First, the topology of its hydrophobic segments is partially inconsistent with sequence-based predictions. This conclusion centers on the presence of two reentrant helices $\left(2^{\text {nd }}\right.$ and $5^{\text {th }}$ as predicted from the sequence) whose two termini are located in the cytosolic side and, as pointed out above, on the presence of one predicted helix $\left(4^{\text {th }}\right)$ that folds as part of the gluzincin domain inside the chamber (as is seen in the Ste24p protease). Second, the open side of the chamber of MecR1 would not reach the outer polar region of the membrane. As a result, several hydrophilic residues of the transmembrane helices and the interior of the chamber are directly exposed to the hydrophobic portion of the membrane (Fig. 1a). This unlikely structure for the transmembrane domain is a consequence of MecR1 having fewer helices, which are shorter and hence would not be able to span the membrane bilayer.

Mapping of the topology of the metalloprotease domain of MecR1. We addressed these two main points through extensive experimental mapping of transmembrane topology. We expressed truncated versions of MecR1, with eGFP fused at the C-terminal of each one of the loops defined between the predicted transmembrane helices, according to Model 1 (Fig. 1b). Our first study was the fluorescence emission in E. coli of these MecR1-eGFP fusion proteins, and also the susceptibility of these proteins to Proteinase K proteolysis in spheroplasts (Fig. 2). eGFP was used as a fluorescent probe of the location of the loops of membrane proteins that are translocated by the Sec system, as is the case for MecR1 (Fig. S3). eGFP is fluorescent when it folds in the cytoplasm but is not fluorescent in the periplasm after its unfolded transport ${ }^{37-39}$. The fluorescence emission of the fusion proteins was measured both in whole cells (Fig. S4) and in membrane protein preparations (Fig. 2a). Expression levels were corroborated by immunodetection (Fig. S5). In agreement with Model 1, the fusions F1 and F5 emitted fluorescence (Fig. 2a) and eGFP was protected from Proteinase K proteolysis (Fig. 2b). These results indicate that eGFP was fused to a cytoplasmic loop of MecR1. Fluorescence microscopy assays showed a homogeneous localization of the fluorescent versions F1 and F5 of MecR1-eGFP in the membrane of $E$. coli (Fig. S6). Although whole cells and membranes expressing F3 showed spectra characteristic of eGFP (Figs. 2a and S4), the intensity was significantly lower in membranes. We interpret this low intensity as the result of proteolysis, with the accumulation of eGFP in the soluble fraction (Fig. S5). Despite the lower fluorescence intensity of F3, protection of eGFP from Proteinase K proteolysis indicated cytoplasmic location. For both F3 and F5, ProtK treatment gave rise to smaller C-terminal fragments that contained eGFP. The mass of the fragments resulting from proteolysis of F3 and F5 (around 35 and $45 \mathrm{kDa}$, respectively) was consistent with proteolysis by ProtK at Thr106, in the extracellular loop that precedes the third TM helix (Fig. S7). The proteins F2C and F6 did not fluoresce and were susceptible to complete degradation by ProtK, indicating that the eGFP domain in these two proteins was located in the periplasm, in agreement with Model 1. The absence of fluorescence emission in F2N and $\mathrm{F} 4 \mathrm{C}$, together with their susceptibility to ProtK, indicated a periplasmic location. This result suggested that Model 1 was incorrect. For F2N, the results suggested that the second hydrophobic region (comprising residues 36-58) would not be structured as a reentrant helix but would instead be a standard transmembrane helix. The fluorescence emission of $\mathrm{F} 4 \mathrm{~N}$ presented a maximum at the wavelength characteristic of eGFP (with low intensity in membranes as was the case for F3; Figs. 2 and S4) and suggested cytoplasmic location. However, unlike the case for F3, F4N was susceptible to ProtK. The accumulation of the $45 \mathrm{kDa}$ C-terminal fragment of F5 in the ProtK assay indicated that, in this longer version, the loops where eGFP was fused in F4N and F4C were indeed located in the cytoplasm, in agreement with Model 1. Hence, the inconsistencies between Model 1 and the assays with F4N and F4C were likely an artifact resulting from the use of truncated versions of MecR1, especially considering that this region is at the core of a folded (gluzincin) domain.

We then used full-length MecR1 to carry out a complementary topology assay using the highly sequence-specific cysteine protease from Tobacco Etch Virus (TEV Protease). Wild-type MecR1 (68.5 kDa, although it runs as $63-65 \mathrm{kDa}$ ) was partially proteolyzed in E. coli, and two C-terminal fragments of approximately $35 \mathrm{kDa}$ and $30 \mathrm{kDa}$ were observed. Mutation of the glutamic residue of the HEXXH metalloprotease motif to Ala (E205A mutation) resulted in the disappearance of the $35 \mathrm{kDa}$ C-terminal fragment of MecR1 (Fig. 3a). Hence, the $35 \mathrm{kDa}$ fragment could be due to auto-proteolysis (which would occur in the absence of antibiotics), as is the case for BlaR $1^{27,40}$. This interpretation assigns a catalytic role to Glu205, as demonstrated for BlaR1. However, we cannot rule out the possibility that the WT and E205A variants differ in their susceptibility to a cellular protease. The $30 \mathrm{kDa}$ fragment accumulated in the mutant protein and thus very likely arose from nonspecific proteolysis by an E. coli protease. Since the presence of this smaller fragment was not detrimental to our study, we used MecR1 E205A in the topology assay. We inserted the recognition site of the TEV Protease in the loops at the positions designated 2N, 2C, 4N, 4C and 5b of MecR1 (Fig. 1b), and we evaluated the susceptibility of these proteins to proteolysis in spheroplasts. We corroborated peptide 36-58 as a membrane-spanning segment, since both $2 \mathrm{~N}$ and $2 \mathrm{C}$ were susceptible to TEV proteolysis as evidenced by the accumulation of $61 \mathrm{kDa}$ and $58 \mathrm{kDa}$ fragments, respectively (Fig. 3b). These results confirm the extracellular location of residues 69-102 (named loop L2 from now onwards). This information was used later to produce an improved structural model (Model 2, Fig. 1c). Constructs $4 \mathrm{~N}, 4 \mathrm{C}$, and $5 \mathrm{~b}$ were not susceptible to TEV proteolysis in spheroplasts, consistent with a cytoplasmic location of these three loops (Fig. 3b). All three constructs were susceptible to TEV proteolysis in membrane preparations, confirming accessibility of the TEV sites in the cytoplasmic loops after cell lysis (Fig. 3c). Interestingly, when the MecR1.E205A.TEV.2C construct was pre-incubated with the $\beta$-lactam antibiotic oxacillin, 
a

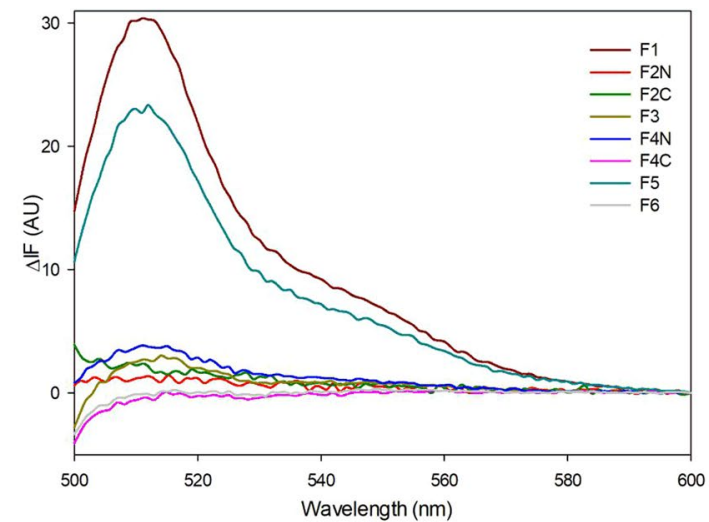

b

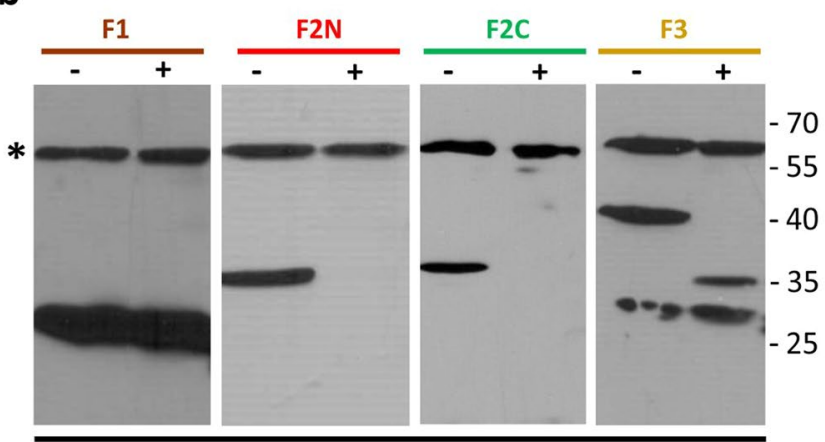

anti-GFP/anti-GroEL

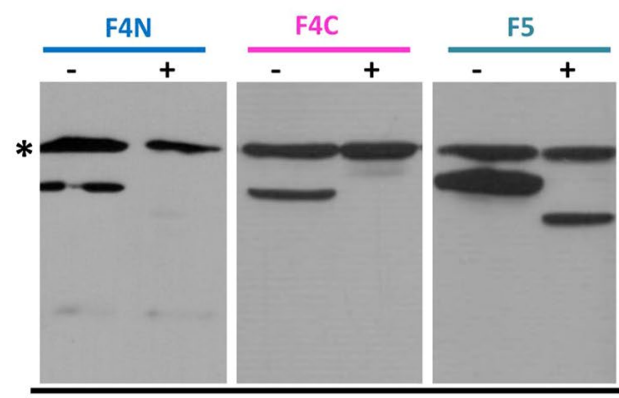

anti-GFP/anti-GroEL

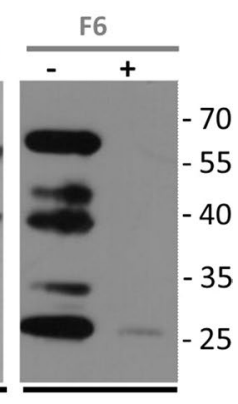

anti-GFP

Figure 2. Topology mapping experiments of MecR1 using truncated fusions to eGFP. (a) Fluorescence emission spectra of each MecR1-eGFP fusion in membrane preparations, after subtraction of the spectra of the membrane proteins from cells harboring the empty vector. (b) Proteinase K protection assay of the MecR1-eGFP fusion proteins. Spheroplasts were incubated in the presence $(+)$ or absence $(-)$ of Proteinase $\mathrm{K}$ to determine the intra- or extracellular location of eGFP. The proteins were immunodetected using a GFP specific antibody. The integrity of the spheroplasts during the Proteinase $\mathrm{K}$ treatment was determined by immunodetection of GroEL (cytoplasmic ${ }^{70,71}$ or inner-membrane associated ${ }^{72}$ ), either simultaneously or sequentially. The GroEL band is indicated with an asterisk. Full, uncut gel images are provided in Fig. S12. Numbers on the right indicate the positions of migration of the molecular mass markers $(\mathrm{kDa})$.

there was no evident proteolysis by TEV protease (Fig. 3d). This was not the case for the other constructs tested. This change in TEV-susceptibility of the C-terminal of loop L2 (where the TEV recognition site is located in construct $2 \mathrm{C}$ ) is consistent with conformational change triggered by the antibiotic.

A key aspect of Model 1, especially evident when aligned to the Ste24p structure relaxed in a membrane, is that the transmembrane domain includes an amphipathic reentrant helix that is inserted in the gluzincin core between the $\mathrm{HE}_{205} \mathrm{LSH}$ and $\mathrm{E}_{245} \mathrm{KVCD}$ putative zinc-binding motifs (Fig. 4a). This reentrant helix (residues 213-243, which turns at $\mathrm{I}^{226} \mathrm{FWFNP}$ ) corresponds roughly to the fifth hydrophobic segment predicted by TopPRED and TMPred (Table 1). Consistent with this aspect of the model, the gluzincin region of the metalloprotease domain of MecR1 E205A (residues 147-304; denominated MecR1 ${ }^{\mathrm{GLZ}}$ ) interacted with the membrane through hydrophobic interactions (Fig. 4b). As expected from the model, we found that MecR1 ${ }^{\mathrm{GLZ}}$ was located in the membrane fraction (M, Fig. 4c). Treatment of membranes with $\mathrm{KCl}$ did not extract MecR1 ${ }^{\mathrm{GLZ}}$ from membranes ${ }^{41}$, thus indicating that it is not a loosely associated peripheral protein (Fig. 4b). The fraction of peripheral proteins associated by strong electrostatic interactions (as probed by $\mathrm{Na}_{2} \mathrm{CO}_{3}$ extraction) did not include MecR $1^{\mathrm{GLZ}}$ either, indicating that it might interact with lipids or proteins in the membrane through hydrophobic interactions. MecR $1^{\mathrm{GLZ}}$ was 
a
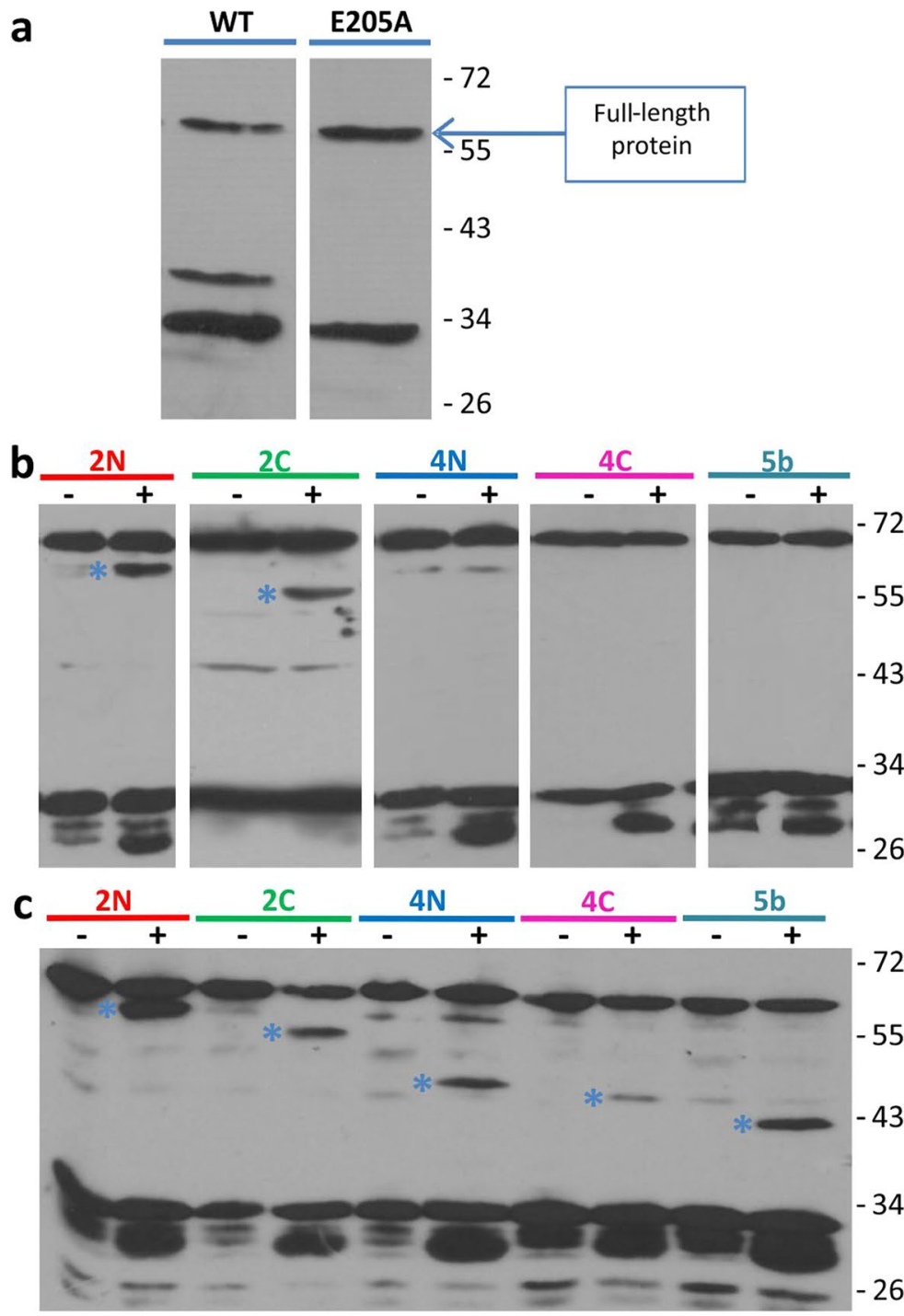

\section{d}

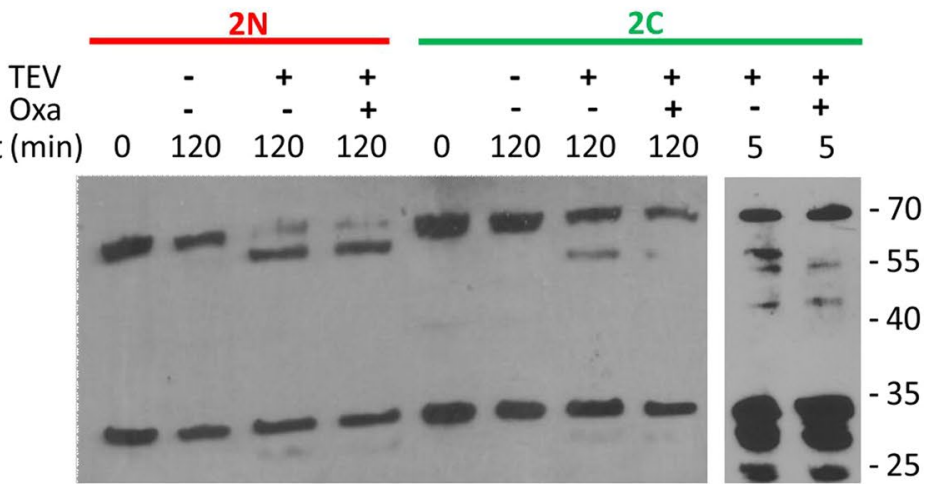

Figure 3. TEV Protease susceptibility assays of the MecR1.E205A.TEV insertional mutant proteins. (a) Expression of full-length MecR1 wild type and the E205A mutant (with a C-terminal His-tag) in E. coli BL21 Star (DE3), evaluated by Western blot using a His-tag specific HRP-conjugated antibody. Full, uncut gel images are provided in Fig. S13. (b) Spheroplasts expressing each MecR1.E205A.TEV insertional mutant protein were incubated in the absence $(-)$ or presence $(+)$ of TEV Protease, in order to determine the intra- or extracellular location of the TEV recognition peptide. (c) Membrane-protein extracts of $E$. coli expressing each MecR1. E205A.TEV insertional mutant protein were prepared and incubated in the absence $(-)$ or presence $(+)$ of TEV protease. In $(\mathbf{b}, \mathbf{c})$, the blue asterisk indicates the $\mathrm{C}$-terminal fragment resulting from TEV proteolysis. The expected molecular weight of the full-length MecR1.E205A.TEV versions is $70.5 \mathrm{kDa}$. The expected molecular weight of the C-terminal His-tagged fragment after TEV treatment for each variant is: $2 \mathrm{~N}, 61.3 \mathrm{kDa} ; 2 \mathrm{C}$, $58.2 \mathrm{kDa} ; 4 \mathrm{~N}, 47.3 \mathrm{kDa} ; 4 \mathrm{C}, 45.3 \mathrm{kDa}$ and $5 \mathrm{~b}, 40.1 \mathrm{kDa}$. (d) Spheroplasts expressing MecR1-TEV-2N and 2C were incubated in the presence $(+)$ or absence $(-)$ of oxacillin, prior to or simultaneously with TEV protease 
treatment. Lanes 2-4 and 6-8: incubation with oxacillin followed by incubation with $750 \mu \mathrm{g} / \mathrm{ml} \mathrm{TEV} \mathrm{protease,}$ $500 \mathrm{rpm}$. Lanes 9 and 10: simultaneous incubation with $100 \mu \mathrm{g} / \mathrm{ml}$ TEV protease, without agitation, as in $B$. Western blots were carried out using a His-tag specific HRP-conjugated antibody. Full, uncut gel images for $B$ and $D$ are provided in Figs. S14 and S15, respectively. Numbers on the right indicate the positions of migration of the molecular weight markers $(\mathrm{kDa})$.

only solubilized from membranes with the detergents C7Bz0, ASB-14, Brij10, Nonidet, and LDAO (Figs. 4c and S8), although most of the protein remained insoluble. Addition of $10 \% \mathrm{~V} / \mathrm{V}$ glycerol improved the amount of soluble MecR $1^{\mathrm{GLZ}}$ recovered from membranes with ASB-14, and MecR1 ${ }^{\mathrm{GLZ}}$ was purified as a folded domain in the presence of detergent (Fig. S9). These results show for the first time that the MecR1 gluzincin core is tailored to be membrane-associated. Therefore, in the initial stages of activation, before the accumulation of MecR2 allows for degradation of MecI by other proteases ${ }^{23}$, MecR1-dependent proteolysis of MecI would require that MecI diffuses to the membrane.

A full-length MecR1 model revealing key interactions between the transmembrane and sensor domains. We corrected Model 1 of the transmembrane domain of MecR1to account for the following key results. Peptide 36-58 is a transmembrane helix instead of a reentrant helix, and loop L2 is extracellular and not a transmembrane helix. We also included in the modeling process the prediction that the L2 loop folds as a partially amphipathic helix upon binding to the sensor domain, a conclusion supported by NMR data for the homologous protein BlaR1 $1^{42}$, and used this information and an X-ray structure of the sensor domain of MecR1 to build a model of the full-length protein. We dubbed this last model Model 2, shown in detail in Fig. 5.

More precisely, we first remodeled residues 1-340 of Model 1 by extending its reentrant helix as a straight transmembrane helix (36-58), spanning the full membrane (Fig. 1c). At this stage of the modeling pipeline we skipped the segment from residue 63 to 103, which includes part of the L2 loop modeled later on. We also stretched the transmembrane helix that connects the metalloprotease and sensor domains (potential helix 6 in Table 1, residues 315 to 338, which Roвetta placed kinked by $90^{\circ}$ at Pro331 leaving the sensor domain right inside the membrane). We shifted the position of this TM helix such that it could help to better close the hydrophilic chamber. The repositioning of this helix in this way leaves, upon connection of the MecR1 sensor domain, the $\beta$-lactam binding site right in front of the C-terminal end of TM helix 2 and the N-terminal end of TM helix 3 , where the connecting L2 loop should locate.

Connection of the transmembrane and sensor domains relied on (i) the finding that the end of the last TM helix (TM6, 315-338) is observed in one of several X-ray structures of the sensor domain of MecR1 (PDB ID 2 IWC ${ }^{43}$, starting at Ser334), and (ii) the NMR-based mapping of the interaction between the L2 loop of BlaR1 and its sensor domain ${ }^{42}$. This NMR study revealed further that L2 binds the sensor domain as an amphipathic alpha helix, through its polar side. The L2 loops of both BlaR1 and MecR1 are short, low complexity peptides, predicted highly disordered. Binding of $\mathrm{L} 2$ in a folded conformation to $\mathrm{BlaR}^{\mathrm{S}}$, as found by the NMR study, resembles the situation found for molecular recognition features in other disordered yet functional regions of proteins ${ }^{44}$. Although the L2 loops of BlaR1 and MecR1 differ in sequence (Fig. S10), they both have amphipathic character. We built an idealized helix from the L2 sequence of MecR1, and docked its polar side to the sensor domain based on the residues of the sensor domain of BlaR1 identified by NMR, transferred from BlaR1 to MecR1. We next placed this $\mathrm{L} 2-\mathrm{MecR}^{\mathrm{S}}$ complex on the revised model of the TM domain (TMD), guided by matching the $\mathrm{N}$-terminal residues of the sensor domain and the last TM helix (TM6). In this operation, the L2 loop in a helical conformation results roughly centered between the $\mathrm{C}$ - and $\mathrm{N}$-terminal ends of TM helices 2 and 3, suggesting that this location is approximately correct. This complex served as a base template for subsequent modeling, including all residues for which we have structural information, located in a way that satisfies sequence continuity and all the known transmembrane topology and interactions between sensor and effector domains. We finally used this TMD-L2-MecR ${ }^{S}$ complex as a template for homology-based modeling, leading to cleaner secondary structures especially with respect to interconnecting loops and turns, while removing strong clashes. Our final model (Model 2) is shown in Fig. 5. This refined model accounts for all of the structural information available from predictions, from existing experimental results, and from our experimental mapping of the topology of the transmembrane domain. The transmembrane domain of MecR1 displays a mostly hydrophobic surface that matches to the hydrophobic portion of the membrane lipids, with a rim of exposed positive residues on the cytoplasmic side and its three tryptophan residues (W40, W113, W228) near the polar lipid heads, as expected for transmembrane proteins ${ }^{45,46}$, and a hydrophilic chamber upholstered with polar residues. Interaction with the sensor domain closes the hydrophilic chamber on the extracellular leaflet of the membrane, leaving mostly polar and charged residues exposed to the extracellular space. The putative site of auto-proteolysis in MecR1, as inferred from results on the homologous protein BlaR1 ${ }^{19,27}$, is close to the active site.

\section{Discussion}

The membrane proteins BlaR1 and MecR1 have not been amenable to overexpression, purification and crystallization. As a result, our understanding of the molecular events that lead to their activation is limited. Our results provide insight to these molecular events. MecR1 has four full transmembrane helices (defined approximately by residues 5-28, 36-58, 110-135, and 315-338) and a reentrant helix (213-243). Both the N- and C-terminus of this helix are located in the cytoplasm, as the helix kinks and turns at the $\mathrm{I}^{226} \mathrm{FWFNP}$ loop. The four transmembrane helices and the reentrant helix form a transmembrane domain. The gluzincin core of the metalloprotease domain (historically known as the cytoplasmic domain) associates with the membrane through hydrophobic interactions. The reentrant helix (residues 213-243) is proposed to anchor this domain. This reentrant helix and 
a

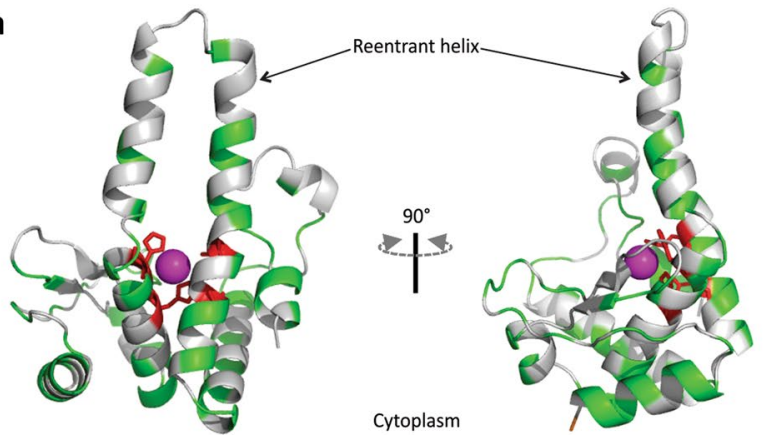

b

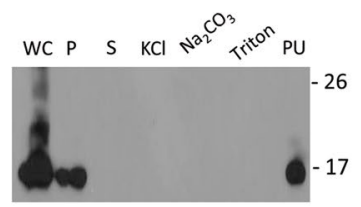

c

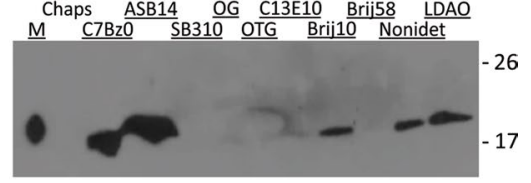

Figure 4. The gluzincin core of MecR1 is membrane-embedded. (a) The modeled gluzincin domain is shown, with hydrophobic residues displayed in light grey, polar residues in green and the putative metal ligands ( $\mathrm{H} 204$,

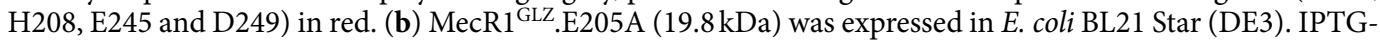
induced cells (WC, whole cell extract) were sonicated and centrifuged to separate the pellet (P; inclusion bodies and large fragments of membrane) from the fraction containing soluble proteins and membrane vesicles. The latter was subjected to ultracentrifugation to separate soluble proteins (S) from membrane proteins. Lanes labelled $\mathrm{KCl}, \mathrm{Na}_{2} \mathrm{CO}_{3}$ and Triton correspond to fractions of proteins solubilized from the membrane preparation with $1 \mathrm{M} \mathrm{KCl}, 0.1 \mathrm{M} \mathrm{Na}_{2} \mathrm{CO}_{3}$ and $0.155 \%$ Triton X-100, respectively. Lane Pu is the pellet remaining after ultracentrifugation of the membranes treated with Triton X-100. (c) Detergent screening to evaluate conditions to solubilize MecR1 ${ }^{\mathrm{GLZ}}$.E205A from membranes. MecR1 ${ }^{\mathrm{GLZ}}$ was found in the membrane protein fraction (M). The fractions corresponding to solubilized proteins are shown. Both the soluble and insoluble fractions are shown in Fig. S8. MecR $1^{\mathrm{GLZ}}$.E205A could only be solubilized from membranes with the detergents C7Bz0, ASB-14, Brij10, Nonidet and LDAO, being the membrane solubilizing zwitterionic detergent ASB-14 (amidosulfobetaine-14) the one that allowed the highest level of protein recovery. In (b,c), MecR1 ${ }^{\text {GLZ }}$.E205A was detected by Western blot using a His-tag specific HRP-conjugated antibody. Numbers on the right indicate the positions of migration of the molecular mass markers $(\mathrm{kDa})$.

the four transmembrane helices define an internal hydrophilic chamber wherein the zinc site sits at its base, as is seen also in the Ste $24 \mathrm{p}$ metalloproteases ${ }^{34}$. Two histidine residues of the $\mathrm{HE}_{205} \mathrm{LSH}$ motif and the glutamic acid of the sequence motif $\mathrm{E}_{245} \mathrm{KVCD}$ would be ligands of the zinc atom, in agreement with results for BlaR1 from B. licheniformis ${ }^{28}$. The fourth ligand would be the hydrolytic water. Our mutagenesis results propose Glu205 as the catalytic base. As is seen in related metalloproteases, Glu205 polarizes the zinc-bound water molecule for nucleophilic attack on the scissile peptide bond, and then transfers a proton to the leaving group of the substrate ${ }^{29,47,48}$. Our proposed zinc ligands in MecR1 differ from those found in minigluzincins, where the $\mathrm{Zn}$ (II) ion is coordinated by the two histidines of the HEXXH motif and by a third histidine ${ }^{49}$.

In our model, the apo-sensor domain effectively seals the hydrophilic chamber, taking the role of the longer and more numerous TM helices of Ste24p-like metalloproteases. The sensor domain lies right above the reentrant helix at the core of the gluzincin domain. This interaction is reinforced through binding of the L2 helix of the metalloprotease domain to the sensor domain, and is in agreement with single-molecule force spectroscopy assays that indicate a strong interaction between the sensor domain and the metalloprotease domain of the homologous protein BlaR1 from B. licheniformis ${ }^{50}$. Two observations lead us to propose that the model shown in Fig. 5 corresponds to the apo-sensor protein (that is, the inactive state prior to interaction with the $\beta$-lactam). First, the site of acylation by the $\beta$-lactam antibiotic is hindered by the binding of the L 2 helix to the sensor domain. Hence, the interaction of the antibiotic with the sensor domain requires dissociation of the helix L2 from the active site groove. Second, the metal site of the metalloprotease domain is too occluded to proteolyze its substrate, MecI, which suggests that our model corresponds to the inactive conformation. The transmembrane architecture of our structural model makes sense given our assumption that the protein is monomeric, as so far is observed for related metalloproteases. This assumption is supported by some of the top predictor models for MecR1, which was released as a mock target for modeling in the $13^{\text {th }}$ edition of the Critical Assessment of Structure Prediction (CASP) (Fig. S11) ${ }^{51}$. However, other CASP models suggest alternative arrangements of the transmembrane helices and gluzincin domain into a half-chamber with its polar residues exposed to the hydrophobic portion of the membrane. This arrangement would only make sense in the context of an oligomer that closes the chamber (Fig. S11).

Our model leads to a proposed mechanism for signal transduction. Acylation of the sensor domain must somehow perturb the metalloprotease domain in order to activate it. How might this proceed? On one hand, NMR studies on S. aureus BlaR1 suggest that acylation of Ser391 alters the dynamics of the sensor domain's core $\beta$ strands, around residues 536 and $563^{52}$. According to our model, these altered regions are within reach of the $\mathrm{I}^{226} \mathrm{FWFNP}$ turn in the reentrant helix of the gluzincin motif, between active site residues $\mathrm{HE}^{205} \mathrm{LSH}$ and $\mathrm{E}^{245} \mathrm{KVCD}$. This interaction is also possible in some of the CASP13 models, consistent with oligomeric arrangements. The connection between the $\beta$-strands of the sensor domain and the $\mathrm{I}^{226} \mathrm{FWFNP}$ turn provides a clear route for transmission of perturbed dynamics, through the reentrant helix directly to the metal site. Based on the observation that serine acylation at the sensor domain of BlaR1 from B. licheniformis detaches it from the L2 loop ${ }^{50,53}$, and our results that indicate a reduction in the susceptibility to proteolysis of the C-terminal end of the $\mathrm{L} 2$ loop in the presence of $\beta$-lactam antibiotics, we propose possible routes for subsequent activation of 

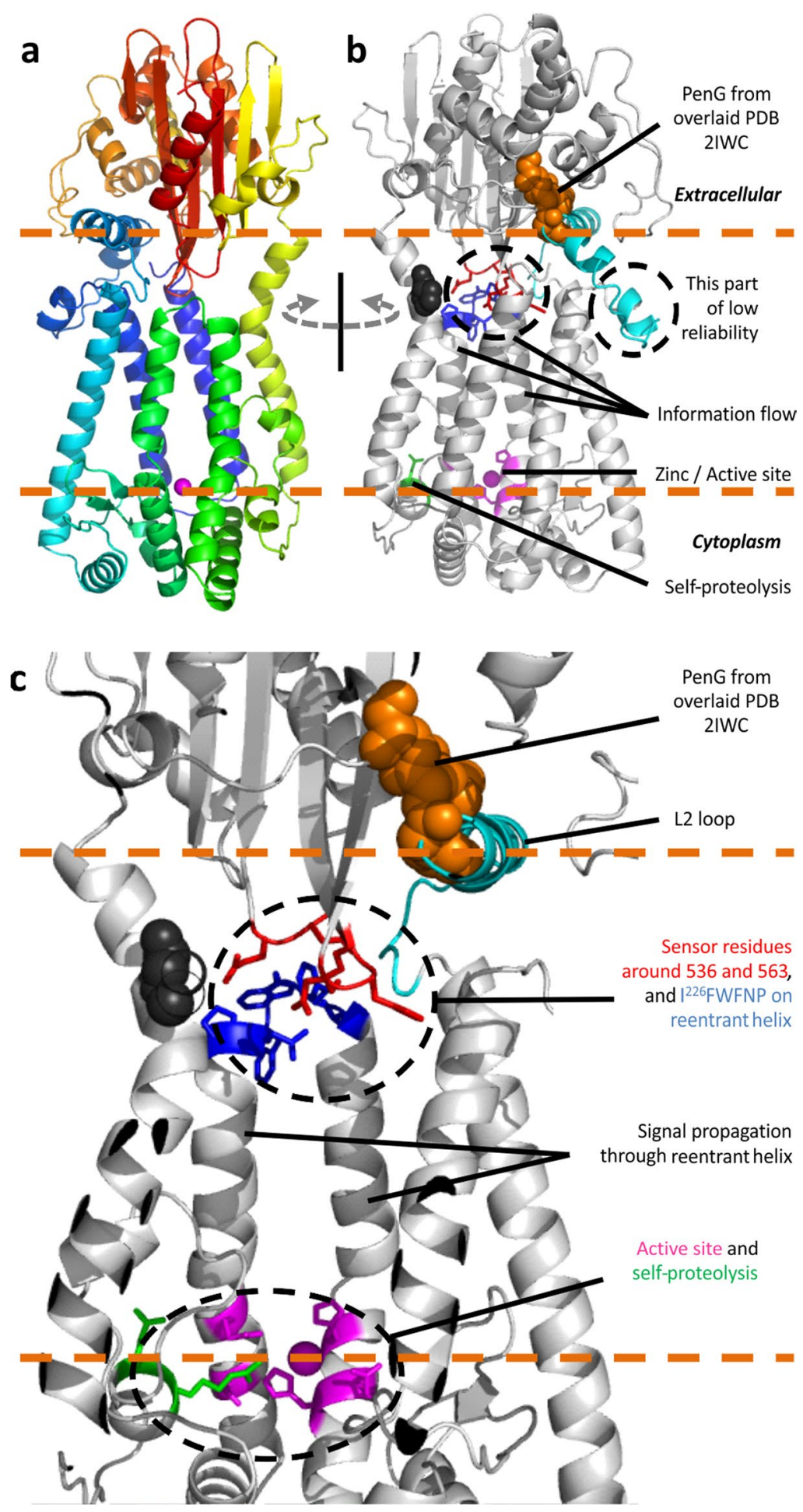

Figure 5. Model of full-length MecR1 (Model 2). (a) The full structure shown as cartoons colored from blue to red (N-terminal to C-terminal). (b) Structure rotated by $180^{\circ}$ around the membrane normal, showing important elements, zoomed in with a cut-through view in (c). The dashed orange lines delineate membrane polar heads that guide protein insertion.

proteolytic action. When the sensor domain detaches from L2 due to steric overlap with the acylated $\beta$-lactam (orange spheres in Fig. 5), it is free to hinge around the end of the last TM helix (possibly around Pro331, and/ or the flexible residues of the N-terminal end of the sensor domain). Dislodgment of the sensor from the hydrophilic chamber will perturb its polar interior, and trigger the requisite conformational change. The nature of such conformational change is yet to be determined, but our model hints at a possible signal transduction mechanism. The transmembrane helices may adjust on the extracellular side so as to hide their internal polar residues from the 
hydrophobic membrane environment, with a concomitant widening and thus opening of the cavity on the cytoplasmic side. The amphipathic L2 helix may also rearrange, likely embedding partially in the membrane in this event as observed in some of the CASP13 models and consistent with higher resistance to proteolysis observed experimentally. Whatever the exact rearrangement, it must end up affecting the local structure around the metal site to enable substrate accessibility and catalysis. Such rearrangement could involve either direct exposure of the gluzincin core towards the cytoplasm, or opening of a crack in the transmembrane domain itself as suggested for ZMPSTE24 $4^{34}$. In conclusion, this study provides the first coherent model of the structure of MecR1, opening routes for future functional investigations on how $\beta$-lactam binding culminates in the proteolytic degradation of MecI.

\section{Methods}

Strains, plasmids and reagents. All chemicals were of the highest quality available from Sigma-Aldrich, BioRad, Thermo Fisher Scientific, Abcam, and Promega. Escherichia coli DH5 $\alpha\left(\mathrm{F}^{-}\right.$甲80lacZ $\Delta \mathrm{M} 15 \Delta$ (lacZYAargF)U169 recA1 endA1 hsd $\mathrm{R} 17\left(\mathrm{r}_{\mathrm{K}}{ }^{-}, \mathrm{m}_{\mathrm{K}}{ }^{+}\right)$phoA supE44 $\lambda^{-}$thi-1 gyrA96 relA1) cells were used for transformation with ligation mixtures for cloning, and for transformation with plasmids for storage as frozen stocks. E. coli BL21 Star (DE3) cells ( $\mathrm{F}^{-}$omp $\mathrm{T}$ hsd $\mathrm{SB}\left(\mathrm{rB}^{-}, \mathrm{mB}^{-}\right) \mathrm{gal} \mathrm{dcm}$ rne131 (DE3)) were employed for protein production from genes cloned in pET24a(+). E. coli MC4100 cells ( $F^{-}$araD139 $\Delta$ (argF-lac) U169 rpsL150 relA1 deoC1 ptsF25 $r p s R$ flbB301 $)^{54}$ were employed for protein production from genes cloned in plasmid pMBLE $^{55}$, under control of a lactose-inducible tac promoter. Two derivatives of strain E. coli MC4100, lacking the two main translocation systems, the secA51 thermosensitive mutant (MC4100; secA51(Ts) leuB::Tn 10) ${ }^{56}$ and $\triangle$ tatC mutant (MC4100; $\Delta t a t C)^{57}$ were also used for protein production from genes cloned in plasmid pMBLE. Luria-Bertani (LB) broth (Difco) was used as growth medium for all bacterial strains.

Genes and oligonucleotides. All synthetic genes, optimized for expression in E. coli, were purchased from Genscript. The mecR1 gene from S. aureus N315 (NRS70) was synthesized with NdeI and BamHI restriction sites for cloning. The egfp gene was ordered with BamHI and HindIII sites for cloning. Oligonucleotides (Table S1) were purchased from either Invitrogen or Genbiotech.

DNA techniques and cloning procedure. DNA preparation and related techniques were performed according to standard protocols ${ }^{58}$. Plasmid DNA was isolated using the Wizard Plus SV Minipreps Kit (Promega). Purification of PCR products and of plasmids from agarose gels was carried out using the Wizard SV Gel and PCR Clean-Up System Kit (Promega). All restriction enzymes were purchased from Promega. T4 DNA Ligase (Promega) and Platinum Pfx DNA Polymerase (Thermo Fisher Scientific) were used according to the user manuals. The sequence of the constructs was verified by the DNA Sequencing Facility at the University of Maine (https://umaine.edu/dnaseq/).

Cloning and expression of the MecR1-eGFP versions. In order to achieve similar levels of expression in the membrane, the corresponding genes were cloned either in the expression vector $\mathrm{pMBLE}^{55}$ or in $\mathrm{pET} 24 \mathrm{a}(+)$, and the proteins were expressed in E. coli MC4100 or BL21 Star (DE3), respectively. The egfp gene was cloned in vector pET24a $(+)$ or in pMBLE between the BamHI and HindIII sites, giving rise to plasmids pET24a(+)::egfp and pMBLE::egfp, respectively. The DNA fragments coding for the different truncated versions of MecR1 were amplified by PCR using the mecR1 synthetic gene as a template, using oLL18 as the forward primer and oLL19, oLL57, oLL20, oLL21, oLL46, oLL22, oLL23, or oLL24 as reverse primers for the mecR1-F1

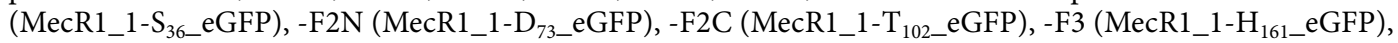

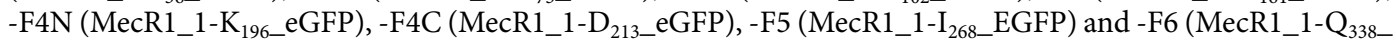
eGFP) constructs, respectively. Each PCR amplification product was isolated, digested with NdeI and BamHI, and finally purified from agarose gels. The fragment was ligated into either the pET24a $(+)::$ egfp or pMBLE::egfp plasmid digested with $\mathrm{NdeI}$ and BamHI. $\mathrm{CaCl}_{2}$-chemically competent $E$. coli $\mathrm{DH} 5 \alpha$ cells were transformed with each ligation mixture. Transformant colonies were selected in LB-kanamycin-agar plates. The constructs designated pMBLE::mecR1-egfp F1 to F6 allowed isopropyl $\beta$-D-1-thiogalactopyranoside (IPTG)-inducible expression of the MecR1 truncated versions fused to the N-terminal of eGFP in E. coli from the tac promoter. E. coli MC4100 cells harboring each pMBLE::mecR1-egfp vector were grown overnight in LB broth supplemented with $50 \mu \mathrm{g} \mathrm{mL}{ }^{-1} \mathrm{kanamycin}$, at $37^{\circ} \mathrm{C}$ and at $220 \mathrm{rpm}$. Fresh medium was inoculated with the overnight culture (dilution of $1 / 100$ ) and grown at $37^{\circ} \mathrm{C}$ until the $\mathrm{OD}_{600}$ reached 0.4 , at which point the cultures were rapidly cooled to $28^{\circ} \mathrm{C}$ and induced with $10 \mu \mathrm{M}$ of IPTG. The cells were grown $4 \mathrm{~h}$ at $28^{\circ} \mathrm{C}$ and at $220 \mathrm{rpm}$. The construct designated pET-24a(+)::mecR1-egfp F6 allowed IPTG-inducible expression of the MecR1 truncated version fused to the N-terminal of eGFP in E. coli from the T7 promoter. E. coli BL21 Star (DE3) cells harboring each the corresponding vector were grown overnight in LB broth supplemented with $50 \mu \mathrm{g} \mathrm{mL}{ }^{-1}$ kanamycin, at $37^{\circ} \mathrm{C}$ and at $220 \mathrm{rpm}$. Fresh medium was inoculated with the overnight culture (dilution of $1 / 100$ ) and grown at $37^{\circ} \mathrm{C}$ until the $\mathrm{OD}_{600}$ reached 0.8 , at which point the cultures were rapidly cooled to $20^{\circ} \mathrm{C}$ and induced with $500 \mu \mathrm{M}$ of IPTG. The cells were grown overnight $(16-20 \mathrm{~h})$ at $20^{\circ} \mathrm{C}$ and at $220 \mathrm{rpm}$.

Cloning and expression of MecR1.E205A and of the MecR1.E205A-TEV insertional mutants. The gene coding for MecR1.E205A was engineered using the overlap extension PCR protocol ${ }^{59}$. The $5^{\prime}$-end of the gene was PCR amplified with the forward primer oLL18 and the reverse mutagenic primer oLL54. The 3 '-end of the mecR1 gene was PCR amplified with the reverse primer oLL26 and the forward mutagenic primer oLL53. Mutagenic primers were designed in order to hybridize to each other. Both PCR reactions were purified using Wizard SV Gel and PCR Clean-Up System kit (Promega) and quantified (Nanodrop 2000, Thermo Fisher Scientific). In a third PCR reaction, equal amounts of both products ( $40 \mathrm{ng}$ ) were used as templates and amplified using external primers oLL18 and oLL26, which introduce an NdeI and an XhoI restriction site, respectively. 
Primer oLL26 was designed without the $m e c R 1$ stop codon, in order to clone the gene in pET24a $(+)$ in phase for the expression of MecR1 E205A with a C-terminal His-tag. The PCR amplification product was purified and digested with NdeI and XhoI restriction enzymes, and purified from agarose gels. The DNA fragment was then ligated into $\mathrm{pET} 24 \mathrm{a}(+)$ digested with the same restriction enzymes. Transformation with the ligation mixture and selection was done as described above.

A sequence coding for the 7 amino acids corresponding to the TEV protease recognition site (ENLYFQG), followed by two flexible amino acids, (GS) was inserted right after the codons coding for amino acids S72 (2N), E101 (2C), D195 (4N), H212 (4C) and I255 (5b) of MecR1 E205A. Insertional mutants 2N, 2C, 4N and 4C were obtained using the overlap extension PCR protocol. External primers used were oLL18 (forward) and oLL26 (reverse). Internal mutagenic primers (reverse primer in the reaction to amplify the $5^{\prime}$-end of the mecR1.E205A gene and forward primer in the reaction to amplify the $3^{\prime}$-end of the gene) were as follows: oLL67 and oLL68 for $2 \mathrm{~N}$; oLL69 and oLL70 for 2C; oLL71 and oLL72 for 4N; oLL73 and oLL74 for $4 \mathrm{C}$. The $5^{\prime}$ end of each mutagenic primer codes for the TEV recognition site. The final PCR amplification product was cloned in pET24a $(+)$, between the NdeI and XhoI sites, as described for mecR1.E205A. A modified protocol was used to generate the gene coding for the $5 \mathrm{~b}$ mutant: the $5^{\prime}$-end of the mutagenic primer codes for the TEV recognition site amino acid sequence and the codons coding for the two flexible amino acids (GS) introduce a BamHI restriction site, which was then used for reconstitution of the gene. One PCR reactions was carried out to amplify the $5^{\prime}$-end of the mecR1.E205A gene using primers oLL18 (forward) and the mutagenic reverse primer oLL90; the amplification product was purified, digested with NdeI and BamHI and cloned in pET24a( $(+)$ digested with the same enzymes. A second PCR reaction was carried out to amplify the $3^{\prime}$-end of mecR1.E205A using the mutagenic forward primer oLL91 and the reverse primer oLL26. The purified PCR fragment was digested with BamHI and XhoI and cloned in pET24a $(+)$, downstream of the $5^{\prime}$-end of mecR1.E205, between the BamHI and XhoI sites. Transformation with the ligation mixture and selection was done as previously described.

MecR1.E205A and the MecR1.E205A-TEV insertional mutants (with a C-terminal His-tag, LEHHHHHH) were expressed in E. coli BL21 Star (DE3) cells harboring the corresponding pET-24a(+) construct. The transformant cells were grown overnight in LB broth supplemented with $50 \mu \mathrm{g} \mathrm{mL}^{-1}$ kanamycin, at $37^{\circ} \mathrm{C}$ and at $220 \mathrm{rpm}$. Fresh medium was inoculated with the overnight culture (dilution of $1 / 100$ ) and grown at $37^{\circ} \mathrm{C}$ until the $\mathrm{OD}_{600}$ reached 0.7 , at which point the cultures were rapidly cooled to $20^{\circ} \mathrm{C}$ and expression of the recombinant proteins was induced with $5 \mu \mathrm{M}$ IPTG at $20^{\circ} \mathrm{C}$ for $16 \mathrm{~h}, 220 \mathrm{rpm}$.

Cloning and expression of MecR1 ${ }^{\mathrm{GLZ}}$. For this construction, the coding DNA fragment was PCR-amplified using the mecR1.E205A gene as the template and primers oLL40 and oLL84, which introduce an NdeI and an XhoI restriction site, respectively. Primer oLL84 was designed without including a stop codon, in order to clone the gene in pET-24a(+) in phase for the expression of with a C-terminal His-tag. The PCR amplification product was purified, digested with NdeI and XhoI, and purified from agarose gels. The DNA fragment was then ligated into $\mathrm{pET}-24 \mathrm{a}(+)$ digested with the same restriction enzymes. Transformation with the ligation mixture and selection was done as previously described. Expression was carried out as described for MecR1.E205A.

Spheroplasts preparation. All steps were performed either in water-ice bath or at $4{ }^{\circ} \mathrm{C}$. The induced culture expressing the protein of interest was cooled down and washed once with Wash Buffer A ( $20 \mathrm{mM} \mathrm{Tris-HCl}$, $\mathrm{pH} 8.0,150 \mathrm{mM} \mathrm{NaCl})$. Next, the cells were pelleted by centrifugation for $6 \mathrm{~min}$ at $2,700 \mathrm{~g}, 4^{\circ} \mathrm{C}$ and resuspended in a volume of Lysozyme Buffer $(20 \mathrm{mM}$ Tris- $\mathrm{HCl}, \mathrm{pH}=8.0,0.1 \mathrm{mM}$ ethylenediaminetetraacetic acid (EDTA), $20 \% \mathrm{w} / \mathrm{v}$ sucrose, $1 \mathrm{mg} \mathrm{mL}^{-1}$ Lysozyme, $0.5 \mathrm{mM}$ phenylmethylsulfonyl fluoride (PMSF)) that was calculated as follow: $\boldsymbol{V}=\left(\mathrm{OD}_{600} *\right.$ initial culture volume $) / 10$. The cells were incubated in this buffer for $30 \mathrm{~min}$ at $4{ }^{\circ} \mathrm{C}$ with gentle rocking at $12 \mathrm{rpm}$. After that, the spheroplasts were pelleted by centrifugation for $4 \mathrm{~min}$ at $2,700 \mathrm{~g}$ and at $4^{\circ} \mathrm{C}$, and resuspended in a volume $\boldsymbol{V}$ of Wash Buffer B $(20 \mathrm{mM}$ Tris- $\mathrm{HCl} \mathrm{pH} 8.0$ buffer, $20 \% \mathrm{w} / \mathrm{v}$ sucrose), pelleted by centrifugation and resuspended in a volume $V$ of Proteolysis Buffer $(20 \mathrm{mM}$ Tris- $\mathrm{HCl} \mathrm{pH} 8.0$ buffer, $10 \mathrm{mM}$ $\mathrm{CaCl}_{2}$ ).

Membrane protein preparations. The cells in $50 \mathrm{~mL}$ of the corresponding induced culture were cooled in ice and harvested by centrifugation for $5 \mathrm{~min}$ at $5,000 \mathrm{~g}, 4^{\circ} \mathrm{C}$. Cells were washed with Buffer A ( $100 \mathrm{mM}$ sodium phosphate, $50 \mathrm{mM}$ sodium bicarbonate, $\mathrm{pH} 7.5$ ) and resuspended in $5 \mathrm{~mL}$ of Buffer A. After addition of $5 \mu \mathrm{M}$ PMSF, the cell contents were liberated by sonification in an ice-water bath (GEX 600 Ultrasonic Processor, Cole-Parmer: 5 cycles of 30 s sonication at $20 \%$ amplitude each, with a $90 \mathrm{~s}$ break in between). The extracts were centrifuged for $20 \mathrm{~min}$ at $5,000 \mathrm{~g}$ and at $4{ }^{\circ} \mathrm{C}$. The pellet from ultracentrifugation of the supernatant $(1 \mathrm{~h}$ at $150,000 \mathrm{~g}$ at $4^{\circ} \mathrm{C}$ ) gave the membrane fraction. This fraction was resuspended in $500 \mu \mathrm{L}$ of Buffer A.

Proteinase K susceptibility assays. The genes coding for fusions F1-F5 were cloned in pMBLE and the proteins were expressed in E. coli MC4100. Due to the lower level of expression of fusion F6, this gene was cloned in pET24a $(+)$ and the protein was expressed in E. coli BL21 Star (DE3). Spheroplasts were suspended in Proteolysis Buffer and incubated on ice for $30 \mathrm{~min}$ without agitation, in the presence (or absence) of $100 \mu \mathrm{g} \mathrm{mL}$ Proteinase K (Sigma). The reaction was stopped by addition of PMSF ( $5 \mathrm{mM}$ final concentration) and centrifugation $(3 \mathrm{~min}$ at $3,200 \mathrm{~g}$ ). The pellet was resuspended in the same volume of Protein Loading Buffer ( $12 \mathrm{mM}$ Tris- $\mathrm{HCl}$ pH 6.8 buffer, 5\% v/v glycerol, 0.4\% SDS, $2.88 \mathrm{mM} 2$-mercaptoethanol, $0.02 \% \mathrm{w} / \mathrm{v}$ bromophenol blue, $5 \mathrm{mM}$ PMSF), and boiled immediately for 5 minutes. Each lane in the SDS-PAGE gel was loaded with spheroplasts corresponding to $2 \times 10^{8}$ cells.

TEV protease susceptibility assays. Tobbaco etch virus (TEV) protease was purified as described by Phan et al..$^{60}$. Spheroplasts prepared from E. coli BL21 Star (DE3) cells harboring the corresponding expression vector were suspended in Proteolysis Buffer and were incubated with $100 \mu \mathrm{g} \mathrm{mL} \mathrm{m}^{-1} \mathrm{TEV}$ protease for $5 \mathrm{~min}$ at $34^{\circ} \mathrm{C}$ 
without agitation. Membrane-protein extracts, from E. coli cells expressing each MecR1.E205A.TEV insertional mutant protein, were prepared and incubated without $(-)$ or with $(+) 500 \mu \mathrm{g} \mathrm{mL}^{-1}$ of TEV protease for $2 \mathrm{~h}$ at $34^{\circ} \mathrm{C}$, and at $1000 \mathrm{rpm}$. Conformational changes triggered by oxacillin were initially evaluated by simultaneous incubation of the antibiotic and the protease. Spheroplasts were incubated for $5 \mathrm{~min}$ at $34^{\circ} \mathrm{C}$ without agitation with $100 \mu \mathrm{g} \mathrm{mL} \mathrm{m}^{-1} \mathrm{TEV}$ protease, in the absence or presence of $455 \mu \mathrm{M}$ oxacillin. In addition, the protease assay was repeated after preincubation with oxacillin. Spheroplasts were prepared and incubated for $15 \mathrm{~min}$ at $37^{\circ} \mathrm{C}$, in the presence (or absence) of $455 \mu \mathrm{M}$ oxacillin. Afterwards the spheroplasts were incubated for $2 \mathrm{~h}$ at $34^{\circ} \mathrm{C}$ and at $500 \mathrm{rpm}$, in the presence (or absence) of $750 \mu \mathrm{g} \mathrm{mL}^{-1}$ of TEV protease. The latter conditions of the protease assay were harsher, in order to assure proteolysis if the TEV recognition site was accessible. In the case of analysis of the proteins in spheroplasts, total protein extracts corresponding to $2 \times 10^{8}$ cells were loaded. In all cases, the reaction was stopped by addition of concentrated Protein Loading Buffer and boiled immediately for $5 \mathrm{~min}$.

Fluorescence spectroscopy. The fluorescence emission spectra of the MecR1-eGFP fusions were recorded with excitation at $\lambda_{\text {exc }}=490 \mathrm{~nm}$ and $\lambda_{\text {em }} 500-600 \mathrm{~nm}$ (Cary Eclipse Fluorescence Spectrophotometer, Agilent). For assays carried out with whole cells suspensions, E. coli cells expressing each one of the different MecR1-eGFP fusions, or transformed with the empty vector, were washed twice with Phosphate buffer $\left(10 \mathrm{mM} \mathrm{NaH}_{2} \mathrm{PO}_{4} \mathrm{pH}\right.$ 7.4 buffer, $280 \mathrm{mM} \mathrm{NaCl}, 6 \mathrm{mM} \mathrm{KCl}$ ). Cells were suspended in an appropriate volume of PBS buffer to give an $\mathrm{OD}_{600}=1$. Membrane protein preparations were carried out as described above for assays carried out with suspensions of membrane vesicles. The amber membrane pellet obtained after ultracentrifugation was resuspended in $500 \mu \mathrm{L}$ of Phosphate buffer and its protein concentration was quantified using the Pierce BCA Protein Assay Kit (Thermo Fisher Scientific). Samples were diluted in PBS to a final concentration of $40 \mu \mathrm{g} \mathrm{mL} \mathrm{L}^{-1}$ of total protein for measurement.

Solubilization of MecR1 ${ }^{\mathrm{GLZ}}$ from $E$. coli membrane preparations. We used a described protocol to sequentially extract membrane proteins ${ }^{41}$. Total membranes were pelleted by ultracentrifugation at $4{ }^{\circ} \mathrm{C}$ for $1 \mathrm{~h}$ at $150,000 \mathrm{~g}$. Membranes were resuspended in $1 \mathrm{M} \mathrm{KCl}$, incubated for $30 \mathrm{~min}$ on ice, and ultracentrifuged to obtain loosely associated peripheral proteins in the supernatant. The peripheral proteins associated by strong electrostatic interactions were released by treatment of the remaining membranes with $0.1 \mathrm{M} \mathrm{Na}_{2} \mathrm{CO}_{3}$ for 30 min on ice followed by ultracentrifugation, to pellet the remaining membranes with the integral or hydrophobically associated proteins. This final pellet was treated with Triton X-100 $(0.155 \% \mathrm{w} / \mathrm{v})$, to extract integral or hydrophobically associated proteins in detergent micelles. Different detergents were evaluated for their capacity to solubilize MecR $1^{\mathrm{GLZ}}$ from membranes. Membranes were prepared as described above. After ultracentrifugation for $1 \mathrm{~h}$ at $150,000 \mathrm{~g}$ and $4{ }^{\circ} \mathrm{C}$, the membranes were suspended using a Potter-Elvehjem PTFE (Sigma-Aldrich) in $1 \mathrm{~mL}$ of $10 \mathrm{mM} \mathrm{NaH}_{2} \mathrm{PO}_{4}, 600 \mathrm{mM} \mathrm{NaCl}, 6 \mathrm{mM} \mathrm{KCl}, 0.1 \mu \mathrm{g} / \mathrm{ml}$ Pefabloc SC (Sigma-Aldrich), pH 7.4 buffer. Aliquots $(100 \mu \mathrm{L})$ were supplemented with detergent $(800 \mu \mathrm{L}$, from Sigma-Aldrich) to reach a final concentration (w/v) of: 3.7\% Chaps, $2 \%$ C7Bz0, 2\% ASB-14, 0.01\% SB3 ${ }_{10}, 7.3 \%$ OG, $0.5 \%$ OTG, $0.5 \% \mathrm{C}_{13} \mathrm{E}_{10}, 0.5 \%$ Brij- $10,0.5 \%$ Brij-58, $0.5 \%$ Nonidet $\mathrm{P} 40$ or $0.5 \%$ LDAO. The resulting suspensions were incubated overnight at $4{ }^{\circ} \mathrm{C}$ with gentle rocking $(12 \mathrm{rpm})$. Subsequently, the samples were centrifuged for $30 \mathrm{~min}$ at $21,000 \mathrm{~g}$ and at $4{ }^{\circ} \mathrm{C}$, to separate the solubilized protein fraction from the pellet of insoluble-precipitated proteins. The effectiveness of each detergent to solubilize the protein was evaluated by SDS-PAGE gel analysis using Western blots (as described below) for detection of protein.

Western blot assays. The protein samples were resolved in 12\% Tris-glycine SDS-PAGE. After electrophoresis, the proteins were transferred to $0.45 \mu \mathrm{M}$ nitrocellulose membranes (BioRad) using Transference Buffer (25 mM Tris, $192 \mathrm{mM}$ Glycine, 20\% Methanol, pH 8.3 buffer) in a Trans-Blot system (BioRad). The membranes were blocked overnight at $4^{\circ} \mathrm{C}$ by incubation with $10 \mathrm{~mL}$ of Blotto $(100 \mathrm{mM}$ Tris- $\mathrm{HCl} \mathrm{pH} 7.5$ buffer, $150 \mathrm{mM}$ $\mathrm{NaCl}, 3 \%$ bovine serum albumin, $3 \%$ non-fat milk, $0.02 \%$ sodium azide). The eGFP fusion proteins were detected by immunoblot analysis using a rabbit GFP-specific primary antibody (ab290, Abcam; 1:10,000 dilution) and a 1:7,000 dilution of the goat anti-rabbit HRP-conjugated secondary antibody (\#32460, Thermo Fisher Scientific). For detection of the control protein GroEL, the membranes were either co-incubated or separately incubated with a rabbit GroEL specific primary antibody ${ }^{61}$ (1:80,000 dilution). The C-terminal His-tag of wild-type MecR1, MecR1.E205A, the MecR1.E205A TEV-insertional mutants, and MecR1 ${ }^{\mathrm{GLZ}}$ were detected by Western bot using a rabbit His-Tag specific antibody conjugated to HRP (ab1187, Abcam; 1: 50,000 dilution). In all cases, the antibodies were diluted in Tween/Tris-buffered saline (T-TBS: $100 \mathrm{mM}$ Tris- $\mathrm{HCl} \mathrm{pH} 7.5$ buffer, $150 \mathrm{mM} \mathrm{NaCl}, 0.1 \%$ Tween 20) with 1.2\% nonfat dry milk. PageRuler Prestained Protein Ladder (Thermo Fisher Scientific) provided the molecular markers. The membranes were washed with T-TBS buffer. The enhanced chemiluminiscence system Super-Signal West Dura Extended Duration Substrate (Pierce) was used according to the manufacturer's instructions to detect the immune complexes, together with CL-XPosure films (Thermo Fisher Scientific).

Molecular modeling and simulations. "Model 1" was the top model delivered by the RoBEtTA server (standard settings as of April 2017) ${ }^{62}$ on MecRl's full sequence. RoBETTA split the protein into 2 large domains connected through the last TM helix. In the main text we discuss the TM domain from this model as the focus of our attention. EVFoLD ${ }^{36}$ (standard settings as of April 2017) computed the evolutionary couplings of the 1-340 segment of MecR1. We consider subsequently a refined MecR1 model, including a TM domain corrected based on our experimental map of the TM topology. The operations for building "Model 2" described in the text were achieved through cycles of manual structure rebuilding in PyMOL, relaxed through atomistic simulations with the AMBER99sBILDN force field in implicit solvent. The resulting basic scaffold was then used as the template for homology modeling with the I-TASSER server ${ }^{63}$. The coordinates of our Model 2 and all 
CASP13-contributed models are available for download and online 3D visualization at http://lucianoabriata. altervista.org/modelshome.html. Membrane-protein systems for molecular dynamics simulations were built with the CHARMM-GUI server as of November $2014^{64}$. The coarse-grained simulation to stabilize ZMPSTE24's structure in a POPE membrane was run with the MARTINI forcefield ${ }^{65}$ in GROMACs $^{66}$. Atomistic simulations with CHARMM $36^{67}$ used parameters for proteins and lipids, and TIP3P ${ }^{68}$ water in NAMD ${ }^{69}$. The protocols provided by the CHARMM-GUI server were used for equilibration and production simulations.

\section{Data availability}

The final structural model of MecR1 generated during the current study, and the MecR1 models obtained from CASP13 structure predictor groups, are available for direct download and online visualization at http:// lucianoabriata.altervista.org/modelshome.html.

Received: 15 February 2019; Accepted: 4 December 2019;

Published online: 20 December 2019

\section{References}

1. Boucher, H. W. Challenges in anti-infective development in the era of bad bugs, no drugs: a regulatory perspective using the example of bloodstream infection as an indication. Clin Infect Dis 50(Suppl 1), S4-9, https://doi.org/10.1086/647937 (2010).

2. Daum, R. S. Community-acquired methicillin-resistant staphylococcus aureus infections. Pediatr Infect Dis J 17, 745-746 (1998).

3. Loffler, C. A. \& Macdougall, C. Update on prevalence and treatment of methicillin-resistant Staphylococcus aureus infections. Expert Rev Anti Infect Ther 5, 961-981, https://doi.org/10.1586/14787210.5.6.961 (2007).

4. Chambers, H. F. Pathogenesis of staphylococcal infection: a manner of expression. J Infect Dis 199, 291-293, https://doi. org/10.1086/595983 (2009).

5. McKenna, M. Vaccine development: Man vs MRSA. Nature 482, 23-25, https://doi.org/10.1038/482023a (2012).

6. Garza-Gonzalez, E. \& Dowzicky, M. J. Changes in Staphylococcus aureus susceptibility across Latin America between 2004 and 2010. Braz J Infect Dis 17, 13-19, https://doi.org/10.1016/j.bjid.2012.08.017 (2013).

7. Turner, N. A. et al. Methicillin-resistant Staphylococcus aureus: an overview of basic and clinical research. Nature reviews. Microbiology 17, 203-218, https://doi.org/10.1038/s41579-018-0147-4 (2019).

8. Poulakou, G., Lagou, S. \& Tsiodras, S. What's new in the epidemiology of skin and soft tissue infections in 2018? Current opinion in infectious diseases 32, 77-86, https://doi.org/10.1097/QCO.0000000000000527 (2019).

9. Livermore, D. M. Has the era of untreatable infections arrived? J Antimicrob Chemother 64(Suppl 1), i29-36, https://doi.org/10.1093/ $\mathrm{jac} / \mathrm{dkp} 255$ (2009).

10. Gilbert, D. N. et al. The ' $10 \times 20$ Initiative': Pursuing a Global Commitment to Develop 10 New Antibacterial Drugs by 2020. Clin. Infect. Dis. 50, 1081-1083, https://doi.org/10.1086/652237 (2010).

11. David, M. Z., Dryden, M., Gottlieb, T., Tattevin, P. \& Gould, I. M. Recently approved antibacterials for methicillin-resistant Staphylococcus aureus (MRSA) and other Gram-positive pathogens: the shock of the new. Int J Antimicrob Agents 50, 303-307, https://doi.org/10.1016/j.ijantimicag.2017.05.006 (2017).

12. Gajdacs, M. The Continuing Threat of Methicillin-Resistant Staphylococcus aureus. Antibiotics 8, https://doi.org/10.3390/ antibiotics8020052 (2019).

13. Talbot, G. H. et al. The Infectious Diseases Society of America's 10 x '20 Initiative (Ten New Systemic Antibacterial Agents FDAapproved by 2020): Is 20 x '20 a Possibility? Clin Infect Dis, ciz089, https://doi.org/10.1093/cid/ciz089 (2019).

14. Banerjee, R., Gretes, M., Basuino, L., Strynadka, N. \& Chambers, H. F. In vitro selection and characterization of ceftobiprole-resistant methicillin-resistant Staphylococcus aureus. Antimicrob Agents Chemother 52, 2089-2096, https://doi.org/10.1128/AAC.01403-07 (2008).

15. Kelley, W. L., Jousselin, A., Barras, C., Lelong, E. \& Renzoni, A. Missense mutations in PBP2A Affecting ceftaroline susceptibility detected in epidemic hospital-acquired methicillin-resistant Staphylococcus aureus clonotypes ST228 and ST247 in Western Switzerland archived since 1998. Antimicrob Agents Chemother 59, 1922-1930, https://doi.org/10.1128/AAC.04068-14 (2015).

16. Chan, L. C. et al. Ceftobiprole- and ceftaroline-resistant methicillin-resistant Staphylococcus aureus. Antimicrob Agents Chemother 59, 2960-2963, https://doi.org/10.1128/AAC.05004-14 (2015).

17. Llarrull, L. I., Fisher, J. F. \& Mobashery, S. Molecular basis and phenotype of methicillin resistance in Staphylococcus aureus and insights into new beta-lactams that meet the challenge. Antimicrob Agents Chemother 53, 4051-4063, https://doi.org/10.1128/ AAC.00084-09 (2009).

18. Peacock, S. J. \& Paterson, G. K. Mechanisms of Methicillin Resistance in Staphylococcus aureus. Annual review of biochemistry 84, 577-601, https://doi.org/10.1146/annurev-biochem-060614-034516 (2015).

19. Llarrull, L. I., Toth, M., Champion, M. M. \& Mobashery, S. Activation of BlaR1 protein of methicillin-resistant Staphylococcus aureus, its proteolytic processing, and recovery from induction of resistance. J Biol Chem 286, 38148-38158, https://doi.org/10.1074/ jbc.M111.288985 (2011).

20. Fuda, C. C., Fisher, J. F. \& Mobashery, S. Beta-lactam resistance in Staphylococcus aureus: the adaptive resistance of a plastic genome. Cell Mol Life Sci 62, 2617-2633, https://doi.org/10.1007/s00018-005-5148-6 (2005).

21. Berger-Bachi, B. Genetic basis of methicillin resistance in Staphylococcus aureus. Cell Mol Life Sci 56, 764-770 (1999).

22. Clarke, S. R. \& Dyke, K. G. The signal transducer (BlaRI) and the repressor (BlaI) of the Staphylococcus aureus beta-lactamase operon are inducible. Microbiology 147, 803-810, https://doi.org/10.1099/00221287-147-4-803 (2001).

23. Arede, P., Milheirico, C., de Lencastre, H. \& Oliveira, D. C. The anti-repressor MecR2 promotes the proteolysis of the mecA repressor and enables optimal expression of beta-lactam resistance in MRSA. PLoS Pathog 8, e1002816, https://doi.org/10.1371/journal. ppat.1002816 (2012).

24. Golemi-Kotra, D., Cha, J. Y., Meroueh, S. O., Vakulenko, S. B. \& Mobashery, S. Resistance to beta-lactam antibiotics and its mediation by the sensor domain of the transmembrane BlaR signaling pathway in Staphylococcus aureus. J Biol Chem 278, 18419-18425, https://doi.org/10.1074/jbc.M300611200 (2003).

25. Cha, J., Vakulenko, S. B. \& Mobashery, S. Characterization of the beta-lactam antibiotic sensor domain of the MecR1 signal sensor/ transducer protein from methicillin-resistant Staphylococcus aureus. Biochemistry 46, 7822-7831, https://doi.org/10.1021/ bi7005459 (2007).

26. Rawlings, N. D. \& Barrett, A. J. Evolutionary families of metallopeptidases. Methods in enzymology 248, 183-228, https://doi. org/10.1016/0076-6879(95)48015-3 (1995).

27. Zhang, H. Z., Hackbarth, C. J., Chansky, K. M. \& Chambers, H. F. A proteolytic transmembrane signaling pathway and resistance to beta-lactams in staphylococci. Science 291, 1962-1965, https://doi.org/10.1126/science.1055144 (2001).

28. Berzigotti, S., Benlafya, K., Sepulchre, J., Amoroso, A. \& Joris, B. Bacillus licheniformis BlaR1 L3 loop is a zinc metalloprotease activated by self-proteolysis. PLoS One 7, e36400, https://doi.org/10.1371/journal.pone.0036400 (2012).

29. Hooper, N. M. Families of zinc metalloproteases. FEBS Lett, 354, 1-6, doi:0014-5793(94)01079-X (1994). 
30. Thumanu, K. et al. Discrete steps in sensing of beta-lactam antibiotics by the BlaR1 protein of the methicillin-resistant Staphylococcus aureus bacterium. Proc. Natl. Acad. Sci. USA 103, 10630-10635, https://doi.org/10.1073/pnas.0601971103 (2006).

31. Wilke, M. S., Hills, T. L., Zhang, H. Z., Chambers, H. F. \& Strynadka, N. C. Crystal structures of the Apo and penicillin-acylated forms of the BlaR1 beta-lactam sensor of Staphylococcus aureus. J. Biol. Chem. 279, 47278-47287, https://doi.org/10.1074/jbc. M407054200 (2004).

32. Borbulevych, O. et al. Lysine Nzeta-decarboxylation switch and activation of the beta-lactam sensor domain of BlaR1 protein of methicillin-resistant Staphylococcus aureus. J. Biol. Chem. 286, 31466-31472, https://doi.org/10.1074/jbc.M111.252189 (2011).

33. Llarrull, L. I., Prorok, M. \& Mobashery, S. Binding of the gene repressor BlaI to the bla operon in methicillin-resistant Staphylococcus aureus. Biochemistry 49, 7975-7977, https://doi.org/10.1021/bi101177a (2010).

34. Quigley, A. et al. The structural basis of ZMPSTE24-dependent laminopathies. Science 339, 1604-1607, https://doi.org/10.1126/ science.1231513 (2013).

35. Pryor, E. E. Jr. et al. Structure of the integral membrane protein CAAX protease Ste24p. Science 339, 1600-1604, https://doi. org/10.1126/science.1232048 (2013)

36. Marks, D. S., Hopf, T. A. \& Sander, C. Protein structure prediction from sequence variation. Nat Biotechnol 30, 1072-1080, https:// doi.org/10.1038/nbt.2419 (2012).

37. Feilmeier, B. J., Iseminger, G., Schroeder, D., Webber, H. \& Phillips, G. J. Green fluorescent protein functions as a reporter for protein localization in Escherichia coli. J Bacteriol 182, 4068-4076, https://doi.org/10.1128/jb.182.14.4068-4076.2000 (2000).

38. Drew, D. et al. Rapid topology mapping of Escherichia coli inner-membrane proteins by prediction and PhoA/GFP fusion analysis. Proc Natl Acad Sci USA 99, 2690-2695, https://doi.org/10.1073/pnas.052018199 (2002).

39. McKenzie, N. L. \& Nodwell, J. R. Transmembrane topology of the AbsA1 sensor kinase of Streptomyces coelicolor. Microbiology 155, 1812-1818, https://doi.org/10.1099/mic.0.028431-0 (2009).

40. Llarrull, L. I. \& Mobashery, S. Dissection of events in the resistance to beta-lactam antibiotics mediated by the protein BlaR1 from Staphylococcus aureus. Biochemistry 51, 4642-4649, https://doi.org/10.1021/bi300429p (2012).

41. Chen, X., Brown, T. \& Tai, P. C. Identification and characterization of protease-resistant SecA fragments: secA has two membraneintegral forms. J Bacteriol 180, 527-537 (1998).

42. Frederick, T. E., Wilson, B. D., Cha, J., Mobashery, S. \& Peng, J. W. Revealing cell-surface intramolecular interactions in the BlaR1 protein of methicillin-resistant Staphylococcus aureus by NMR spectroscopy. Biochemistry 53, 10-12, https://doi.org/10.1021/ bi401552j (2014)

43. Marrero, A., Mallorqui-Fernandez, G., Guevara, T., Garcia-Castellanos, R. \& Gomis-Ruth, F. X. Unbound and acylated structures of the MecR1 extracellular antibiotic-sensor domain provide insights into the signal-transduction system that triggers methicillin resistance. J Mol Biol 361, 506-521, https://doi.org/10.1016/j.jmb.2006.06.046 (2006).

44. Mohan, A. et al. Analysis of molecular recognition features (MoRFs). J Mol Biol 362, 1043-1059, https://doi.org/10.1016/j. jmb.2006.07.087 (2006)

45. Ulmschneider, M. B. \& Sansom, M. S. Amino acid distributions in integral membrane protein structures. Biochim Biophys Acta 1512, 1-14, https://doi.org/10.1016/s0005-2736(01)00299-1 (2001).

46. Pilpel, Y., Ben-Tal, N. \& Lancet, D. kPROT: a knowledge-based scale for the propensity of residue orientation in transmembrane segments. Application to membrane protein structure prediction. J Mol Biol 294, 921-935, https://doi.org/10.1006/jmbi.1999.3257 (1999).

47. Gao, X. et al. Structural basis for the autoprocessing of zinc metalloproteases in the thermolysin family. Proc Natl Acad Sci USA 107, 17569-17574, https://doi.org/10.1073/pnas.1005681107 (2010).

48. Le Moual, H., Devault, A., Roques, B. P., Crine, P. \& Boileau, G. Identification of glutamic acid 646 as a zinc-coordinating residue in endopeptidase-24.11. J Biol Chem 266, 15670-15674 (1991).

49. Lopez-Pelegrin, M. et al. A novel family of soluble minimal scaffolds provides structural insight into the catalytic domains of integral membrane metallopeptidases. J Biol Chem 288, 21279-21294, https://doi.org/10.1074/jbc.M113.476580 (2013)

50. Mescola, A., Dauvin, M., Amoroso, A., Duwez, A. S. \& Joris, B. Single-molecule force spectroscopy to decipher the early signalling step in membrane-bound penicillin receptors embedded into a lipid bilayer. Nanoscale 11, 12275-12284, https://doi.org/10.1039/ c9nr02466b (2019).

51. Abriata, L. A., Tamo, G. E. \& Dal Peraro, M. A further leap of improvement in tertiary structure prediction in CASP13 prompts new routes for future assessments. Proteins. https://doi.org/10.1002/prot.25787 (2019).

52. Staude, M. W. et al. Investigation of signal transduction routes within the sensor/transducer protein BlaR1 of Staphylococcus aureus. Biochemistry 54, 1600-1610, https://doi.org/10.1021/bi501463k (2015).

53. Hanique, S. et al. Evidence of an intramolecular interaction between the two domains of the BlaR1 penicillin receptor during the signal transduction. J Biol Chem 279, 14264-14272, https://doi.org/10.1074/jbc.M313488200 (2004).

54. Checa, S. K. \& Viale, A. M. The 70-kDa heat-shock protein/DnaK chaperone system is required for the productive folding of ribulose-biphosphate carboxylase subunits in Escherichia coli. Eur J Biochem 248, 848-855, https://doi.org/10.1111/j.14321033.1997.00848.x (1997).

55. Gonzalez, L. J. et al. Membrane anchoring stabilizes and favors secretion of New Delhi metallo-beta-lactamase. Nat Chem Biol 12, 516-522, https://doi.org/10.1038/nchembio.2083 (2016)

56. Bowers, C. W., Lau, F. \& Silhavy, T. J. Secretion of LamB-LacZ by the signal recognition particle pathway of Escherichia coli. J Bacteriol 185, 5697-5705, https://doi.org/10.1128/jb.185.19.5697-5705.2003 (2003).

57. Bogsch, E. G. et al. An essential component of a novel bacterial protein export system with homologues in plastids and mitochondria. J Biol Chem 273, 18003-18006, https://doi.org/10.1074/jbc.273.29.18003 (1998).

58. Sambrook, J., Fritsch, E. F. \& Maniatis, T. Molecular Cloning: A Laboratory Manual. 2nd edn, (1989).

59. Bryksin, A. \& Matsumura, I. Overlap extension PCR cloning. Methods Mol Biol 1073, 31-42, https://doi.org/10.1007/978-1-62703625-2 4 (2013)

60. Phan, J. et al. Structural basis for the substrate specificity of tobacco etch virus protease. J Biol Chem 277, 50564-50572, https://doi. org/10.1074/jbc.M207224200 (2002).

61. Moran-Barrio, J., Limansky, A. S. \& Viale, A. M. Secretion of GOB metallo-beta-lactamase in Escherichia coli depends strictly on the cooperation between the cytoplasmic DnaK chaperone system and the Sec machinery: completion of folding and $\mathrm{Zn}$ (II) ion acquisition occur in the bacterial periplasm. Antimicrob Agents Chemother 53, 2908-2917, https://doi.org/10.1128/AAC.01637-08 (2009).

62. Kim, D. E., Chivian, D. \& Baker, D. Protein structure prediction and analysis using the Robetta server. Nucleic Acids Res 32, W526-531, https://doi.org/10.1093/nar/gkh468 (2004).

63. Zhang, Y. I-TASSER server for protein 3D structure prediction. BMC Bioinformatics 9, 40, https://doi.org/10.1186/1471-2105-9-40 (2008).

64. Jo, S., Kim, T., Iyer, V. G. \& Im, W. CHARMM-GUI: a web-based graphical user interface for CHARMM. J Comput Chem 29, 1859-1865, https://doi.org/10.1002/jcc.20945 (2008).

65. Marrink, S. J., Risselada, H. J., Yefimov, S., Tieleman, D. P. \& de Vries, A. H. The MARTINI force field: coarse grained model for biomolecular simulations. J Phys Chem B 111, 7812-7824, https://doi.org/10.1021/jp071097f (2007).

66. Abraham, M. J. et al. GROMACS: High performance molecular simulations through multi-level parallelism from laptops to supercomputers. SoftwareX 1-2, 19-25, https://doi.org/10.1016/j.softx.2015.06.001 (2015). 
67. Best, R. B. et al. Optimization of the additive CHARMM all-atom protein force field targeting improved sampling of the backbone phi, psi and side-chain chi(1) and chi(2) dihedral angles. J Chem Theory Comput 8, 3257-3273, https://doi.org/10.1021/ct300400x (2012).

68. Jorgensen, W. L., Chandrasekhar, J., Madura, J. D., Impey, R. W. \& Klein, M. L. Comparison of simple potential functions for simulating liquid water. The Journal of Chemical Physics 79, 926-935, https://doi.org/10.1063/1.445869 (1983).

69. Phillips, J. C. et al. Scalable molecular dynamics with NAMD. J Comput Chem 26, 1781-1802, https://doi.org/10.1002/jcc.20289 (2005).

70. DeLisa, M. P., Tullman, D. \& Georgiou, G. Folding quality control in the export of proteins by the bacterial twin-arginine translocation pathway. Proc Natl Acad Sci USA 100, 6115-6120, https://doi.org/10.1073/pnas.0937838100 (2003).

71. Marrichi, M., Camacho, L., Russell, D. G. \& DeLisa, M. P. Genetic toggling of alkaline phosphatase folding reveals signal peptides for all major modes of transport across the inner membrane of bacteria. J Biol Chem 283, 35223-35235, https://doi.org/10.1074/jbc. M802660200 (2008).

72. Torok, Z. et al. Evidence for a lipochaperonin: association of active protein-folding GroESL oligomers with lipids can stabilize membranes under heat shock conditions. Proc Natl Acad Sci USA 94, 2192-2197, https://doi.org/10.1073/pnas.94.6.2192 (1997).

\section{Acknowledgements}

B.S.B., E.G. and D.M. are Ph.D. Fellows of CONICET. L.I.L. is a Staff member of CONICET. This work was supported by grants from Agencia Nacional de Promoción Científica y Tecnológica (ANPCyT) to L.I.L (PICT2011-0949, PICT-2015-2521). L.I.L. was a Pew Latin American Fellow in the Biomedical Sciences and this work was supported in part by The Pew Charitable Trusts. The opinions expressed are those of the authors and do not necessarily reflect the views of The Pew Charitable Trusts. We thank Dr. Viale (IBR, CONICET-UNR) for kindly providing the GroEL-specific antibody and the MC4100 derived strains. We thank the CASP13 organizers and predictors for access to the CASP13 models. We thank Dr. Jed Fisher (University of Notre Dame) for insightful discussion on the manuscript.

\section{Author contributions}

B.S.B., L.A.A. and E.G. contributed equally to this work. Conceptualization, L.I.L; Methodology, L.I.L., L.A.A., E.G. and B.S.B.; Investigation, L.I.L. and L.A.A.; Research design, L.I.L. and L.A.A.; B.S.B., L.A.A., E.G. and D.M. performed research; M.D.P. contributed to molecular modeling; Writing of original draft, B.S.B., L.A.A., M.D.P. and L.I.L.; Review and Editing, E.G., L.A.A. and L.I.L.; Funding Acquisition and Supervision, L.I.L.; Computational Resources, M.D.P.

\section{Competing interests}

The authors declare no competing interests.

\section{Additional information}

Supplementary information is available for this paper at https://doi.org/10.1038/s41598-019-55923-z.

Correspondence and requests for materials should be addressed to L.I.L.

Reprints and permissions information is available at www.nature.com/reprints.

Publisher's note Springer Nature remains neutral with regard to jurisdictional claims in published maps and institutional affiliations.

(c) (i) Open Access This article is licensed under a Creative Commons Attribution 4.0 International

License, which permits use, sharing, adaptation, distribution and reproduction in any medium or format, as long as you give appropriate credit to the original author(s) and the source, provide a link to the Creative Commons license, and indicate if changes were made. The images or other third party material in this article are included in the article's Creative Commons license, unless indicated otherwise in a credit line to the material. If material is not included in the article's Creative Commons license and your intended use is not permitted by statutory regulation or exceeds the permitted use, you will need to obtain permission directly from the copyright holder. To view a copy of this license, visit http://creativecommons.org/licenses/by/4.0/.

(C) The Author(s) 2019 